\title{
Complexity Measures from Interaction Structures
}

\author{
T. Kahle ${ }^{1}$, E. Olbrich ${ }^{1}$, J. Jost ${ }^{1,2}$, and N. Ay ${ }^{1,2}$ \\ 1 Max Planck Institute for Mathematics in the Sciences, \\ Inselstrasse 22, D-04103 Leipzig, Germany and \\ 2 Santa Fe Institute, 1399 Hyde Park Road, Santa Fe, NM 87501, USA
}

(Dated: August 8, 2018)

\begin{abstract}
We evaluate information theoretic quantities that quantify complexity in terms of $k$-th order statistical dependencies that cannot be reduced to interactions among $k-1$ random variables. Using symbolic dynamics of coupled maps and cellular automata as model systems, we demonstrate that these measures are able to identify complex dynamical regimes.
\end{abstract}

PACS numbers: 89.70.Cf, 89.75.Fb, 89.75.Kd

\section{INTRODUCTION}

In this paper we study a notion of complexity based on interaction among parts of a system. This is one of the first and most natural choices and has been considered several times. Doing so, we will pursue a geometric approach going back to the works of Amari [1, 2]. In statistical physics, for example, the complexity of a model is specified by the order of interaction. Firstly, one considers the free theory with no interaction, and as the next approximation a theory of pair interaction. That means that there exists an energy function $H$, assigning to each state of the world $x$ a real number $H(x)$ such that the more probable states have a lower energy. If this function has, using a suitable expansion into polynomials, only terms of order two, one speaks of pair interaction. The probability to find the world in the state $x$ at temperature $T$ is given by the Gibbs distribution

$$
P(x)=\frac{\mathrm{e}^{-\beta H(x)}}{Z(\beta)},
$$

where, $k$ denoting Boltzmann's constant, $\beta$ is the inverse temperature $\frac{1}{k T}$. By

$$
Z(\beta):=\sum_{x} \mathrm{e}^{-\beta H(x)}
$$

we denoted the partition function, which is a normalization.

In this work, we study a complexity measure that incorporates the idea to quantify the amount of pairwise, triple wise,... -interaction in a rigorous way. We do so by using the exponential families of $k$-interactions which will be defined in Section II. In this context, a $k$-interaction is an interaction between $k$ random variables. They can stand for $k$ different particles in the spatial case, one variable in $k$ time steps, or a mixture of both. Using the notion of distance from such an exponential family, we can quantify the amount of $k$-wise interaction that cannot be explained by $(k-1)$-wise interaction. This allows

*Electronic address: kahle@mis.mpg.de us to quantify complexity. We explore this concept using two examples: Symbolic Dynamics of Coupled Map Lattices and Cellular Automata. For coupled map lattices we focus on spatial interaction. Still, the temporal and spatiotemporal features can be studied analogously as we will see for Cellular Automata. Our approach quantifies all interactions in a system. It does not necessarily refer to any neighborhood structure, any possible subsystem can contribute to this interaction. Allowing every subsystem, not necessarily local, to interact is motivated by possible applications as for example the identification of positions in the genome that carry a high information content. Interacting regions in the genome are not necessarily located spatially close to each other. For example the arrangement of the chromosome in space can bring sequentially distant sites into spatial proximity and thereby enable regulatory interactions.

The paper is organized as follows. In Section II we introduce the necessary concepts from information theory and define our model systems. We give details on the models and numerical methods in Section III and in Section IV we give the results. Section $\mathrm{V}$ contains concluding remarks.

\section{INFORMATION THEORY}

In this section we introduce exponential families of probability measures, the Kullback-Leibler divergence and other notions. Throughout the whole paper, the set of states of the system is of compositional structure. That means, for each site index $v$ in a finite set $V:=\{1, \ldots, N\}$ we have a finite configuration space $\mathcal{X}_{v}$. The set of all possible configurations is

$$
\mathcal{X}_{V}:=\underset{v \in V}{\times} \mathcal{X}_{v}
$$

and likewise for each subset $A \subseteq V, \mathcal{X}_{A}:=\times_{v \in A} \mathcal{X}_{v}$. Every real valued function on the set $\mathcal{X}_{V}$ can be seen as an element of the vector space

$$
\mathbb{R}^{\mathcal{X}_{V}}:=\left\{f: \mathcal{X}_{V} \rightarrow \mathbb{R}\right\}
$$

As $\left|\mathcal{X}_{V}\right|$ is finite, this space is isomorphic to $\mathbb{R}^{n}$ with $n=\left|\mathcal{X}_{V}\right|$. We will therefore call elements in $\mathbb{R}^{\mathcal{X}_{V}}$ vectors 
or functions without preference.

\section{A. Families of Probability Measures}

We now study probability distributions on the set $\mathcal{X}_{V}$. Naturally, these are also elements of $\mathbb{R}^{\mathcal{X}_{V}}$. Consider

$$
\overline{\mathcal{P}\left(\mathcal{X}_{V}\right)}:=\left\{P \in \mathbb{R}^{\mathcal{X}_{V}}: P(x) \geq 0, \sum_{x \in \mathcal{X}_{V}} P(x)=1\right\},
$$

the probability measures on the set $\mathcal{X}_{V}$. Due to the compositional structure these probability measures are in fact only joint probabilities of a set of random variables $\left\{X_{v}: v \in V\right\}$, where $X_{v}$ takes values in $\mathcal{X}_{v} \cdot \overline{\mathcal{P}\left(\mathcal{X}_{V}\right)}$ has the geometrical structure of a $\left(\left|\mathcal{X}_{V}\right|-1\right)$-dimensional simplex. For $P \in \overline{\mathcal{P}\left(\mathcal{X}_{V}\right)}$, we call

$$
\operatorname{supp}(P):=\left\{x \in \mathcal{X}_{V}: P(x)>0\right\}
$$

the support of $P$. Then the probability distributions with full support are denoted

$$
\mathcal{P}\left(\mathcal{X}_{V}\right):=\left\{P \in \overline{\mathcal{P}\left(\mathcal{X}_{V}\right)}: P(x)>0, \forall x \in \mathcal{X}_{V}\right\} .
$$

The notation is justified as $\overline{\mathcal{P}\left(\mathcal{X}_{V}\right)}$ is the closure of $\mathcal{P}\left(\mathcal{X}_{V}\right)$.

Example 1. We consider the example $V=\{1,2\}$ in the binary case, i.e. $\mathcal{X}_{1}=\mathcal{X}_{2}=\{0,1\}$. We have $\mathcal{X}_{V}=$ $\{0,1\}^{2}=\{(00),(01),(10),(11)\}$, and $\mathbb{R}^{\mathcal{X}_{V}}$ is just $\mathbb{R}^{4}$. The set of probability measures $\overline{\mathcal{P}\left(\mathcal{X}_{V}\right)}$ is a three-dimensional tetrahedron

$$
\left\{\left(p_{00}, p_{01}, p_{10}, p_{11}\right) \in \mathbb{R}_{\geq 0}^{4}: p_{11}=1-p_{00}-p_{01}-p_{10}\right\} .
$$

It is the set of joint probabilities of two binary random variables. The extreme points of this tetrahedron are the 4 unit vectors.

For two distributions $P, Q \in \overline{\mathcal{P}\left(\mathcal{X}_{V}\right)}$, we can now define a notion of distance by

$$
D(P \| Q):= \begin{cases}\sum_{x \in \mathcal{X}} P(x) \log _{2} \frac{P(x)}{Q(x)} & \text { if }(*) \text { holds } \\ \infty & \text { otherwise. }\end{cases}
$$

Here, $(*)$ is the condition $\operatorname{supp}(P) \subseteq \operatorname{supp}(Q) . D(P \|$ $Q)$ is called the Kullback-Leibler divergence or relative entropy. Although not a metric, it is non-negative and equals zero if and only if $P \equiv Q$.

In information geometry one studies families of probability measures i.e. sub manifolds of $\overline{\mathcal{P}\left(\mathcal{X}_{V}\right)}$. A very natural class of such families arises if we consider the exponential map

$$
\exp : \mathbb{R}^{\mathcal{X}_{V}} \rightarrow \mathcal{P}\left(\mathcal{X}_{V}\right) \quad f \mapsto \frac{\mathrm{e}^{f}}{\sum_{x \in \mathcal{X}_{V}} \mathrm{e}^{f(x)}} .
$$

It acts by component-wise exponentiation and normalization.
Definition 2. Let $\mathcal{I}$ be a linear subspace of $\mathbb{R}^{\mathcal{X}_{V}}$. We define the exponential family $\mathcal{E}_{\mathcal{I}}$ to be the image of $\mathcal{I}$ under the exponential map:

$$
\mathcal{E}_{\mathcal{I}}:=\exp (\mathcal{I})
$$

Exponential families are well known in statistical science. They have many nice properties with respect to maximum likelihood estimation and other inference methods. For our application we are, for a given $P$, interested in minimizing the distance $D(P \| Q)$ for $Q$ in a given exponential family. It can be seen, that this is equivalent to maximum likelihood estimation of $P$ in the family $\mathcal{E}$. To do so, let $\hat{P}$ be the relative frequencies of an observation. Then the log-likelihood function with respect to $Q$ is $L(\hat{P}, Q):=\sum_{x \in \mathcal{X}_{V}} \hat{P}(x) \log Q(x)$. Maximum likelihood estimation is to find a $Q$ that maximizes this function. On the other hand, if we add to $-L$ the constant (with respect to $Q$ ) $\sum_{x \in \mathcal{X}_{V}} \hat{P}(x) \log \hat{P}(x)$ we find

$$
\begin{aligned}
-L(\hat{P}, Q) & +\sum_{x \in \mathcal{X}_{V}} \hat{P}(x) \log \hat{P}(x) \\
& =\sum_{x \in \mathcal{X}_{V}} \hat{P}(x) \log \frac{\hat{P}(x)}{Q(x)} \\
& =D(\hat{P} \| Q)
\end{aligned}
$$

Therefore a maximizer of the likelihood is a minimizer of Kullback-Leibler distance. On the other hand, geometrically, minimization of $D(P \| Q)$ could be interpreted as a projection, since $D$ is a kind of distance. This view is employed in information geometry. These projections are the key players in the definition of our complexity measure.

In what follows, we define the concrete exponential families that we will use in the definition of our measure. The idea that we want to follow was first explored by Darroch et al. [3]. It is to consider exponential families of $k$ interactions. Firstly, one defines the linear space of functions depending on only $k$ of their arguments. After taking the exponential map of sums of such functions, we find probability distributions with only $k$-interactions. For a given probability distribution, coming from a real system, we then want to quantify its complexity by measuring how far it is from being reducible to a theory of $k$-interactions.

\section{B. Interaction Spaces}

We exploit the compositional structure of $\mathcal{X}_{V}$ to define the notion of $k$-th order interactions.

For any given $A \subseteq V$, we write $x \in \mathcal{X}_{V}$ as $x=$ $\left(x_{A}, x_{V \backslash A}\right)$ i.e. we distinguish between components in $A$ and outside $A$. Then we define $\mathcal{I}_{A}$ to be the subspace of functions that do not depend on the configurations 
outside $A$ :

$$
\begin{aligned}
\mathcal{I}_{A}:= & \left\{f \in \mathbb{R}_{V}^{\mathcal{X}}: f\left(x_{A}, x_{V \backslash A}\right)=f\left(x_{A}, x_{V \backslash A}^{\prime}\right)\right. \\
& \text { for all } \left.x_{A} \in \mathcal{X}_{A}, x_{V \backslash A}, x_{V \backslash A}^{\prime} \in \mathcal{X}_{V \backslash A}\right\} .
\end{aligned}
$$

Using these spaces as building blocks, one can define the interaction space corresponding to interactions between $k$ arbitrary units

$$
\mathcal{I}_{k}:=\operatorname{span}_{A \subseteq V,|A|=k} \mathcal{I}_{A},
$$

i.e. just the span of all the vectors on the right hand side. Associated with each of the interaction spaces is an exponential family $\mathcal{E}_{k}:=\exp \left(\mathcal{I}_{k}\right)$. The interaction spaces are included in each other: $\mathcal{I}_{1} \subseteq \mathcal{I}_{2} \subseteq \cdots \subseteq \mathcal{I}_{N}$, therefore we have defined a hierarchy of exponential families:

$$
\mathcal{E}_{1} \subseteq \mathcal{E}_{2} \subseteq \cdots \subseteq \mathcal{E}_{N} .
$$

This hierarchy was studied in $[1,4]$. It has found various applications in the theory of neural networks. The correspondence with the notions of statistical physics is as follows: A vector $f \in \mathcal{I}_{k}$ corresponds to an energy which has only $k$-interactions, but no higher interactions. It gives rise to a probability distribution

$$
P(x)=\frac{\mathrm{e}^{f(x)}}{Z} \in \mathcal{E}_{k} .
$$

Vice versa every $P$ in, for example, $\mathcal{E}_{2}$ has a (non-unique) representation as

$$
\begin{aligned}
P(x) & =\prod_{A \subseteq V:|A|=2} \phi_{A}(x) \\
& =\frac{1}{Z} \exp \left(\sum_{A \subseteq V:|A|=2} f_{A}\left(x_{A}\right)\right)
\end{aligned}
$$

where the energy can be written with pair interactions only.

Note that $\mathcal{E}_{N}$, corresponding to all functions, is $\mathcal{P}\left(\mathcal{X}_{V}\right)$. In general, in the image of the exponential map, one has only distributions with full support. Probability zero corresponds to infinite energy which is only achievable by limits of sequences of probability measures in an exponential family. It is an open question which of the possible support sets are achievable by such limit probability measures in a given exponential family. This question is connected with the face structure of a convex polytope, the so called marginal polytope. See [5, 6] for details. For our application one can just pass to the closure(in $\mathbb{R}^{n}$ ) of the exponential family. In practice, if $\hat{P}$ is the distribution given by the relative frequencies observed in an experiment, we can compute the minimizer of the Kullback-Leibler distance to an exponential family using the iterative proportional fitting algorithm from [7]. However, the complexity of the algorithm makes it unfeasible for values of $\left|\mathcal{X}_{V}\right| \gg 10^{6}$.
Now we are about to introduce our complexity measures. They are based on the notion of distance to an exponential family $\mathcal{E}$. We define

$$
D(P \| \mathcal{E}):=\inf _{Q \in \mathcal{E}} D(P \| Q) .
$$

One can show that $D(P \| \mathcal{E})$ is continuous with respect to $P \in \overline{\mathcal{P}\left(\mathcal{X}_{V}\right)}$. Given two exponential families $\mathcal{E} \subseteq \mathcal{F}$ on has

$$
D(P \| \mathcal{E})-D(P \| \mathcal{F}) \geq 0 .
$$

We now use differences like these as components of a vector valued complexity measure.

\section{Complexity measures}

\section{Definition}

For a given $P \in \overline{\mathcal{P}\left(\mathcal{X}_{V}\right)}$, we define a vector valued complexity measure $I(P):=\left(I^{(1)}(P), \ldots, I^{(N)}(P)\right)$ with components

$$
I^{(k)}(P):=D\left(P \| \mathcal{E}_{k-1}\right)-D\left(P \| \mathcal{E}_{k}\right), \quad k=1, \ldots, N .
$$

In the next section we will argue for the choice and discuss properties.

\section{Properties and Interpretation}

The main idea is that $I^{(k)}$ quantifies those dependencies between $k$ nodes that are not captured by interactions between smaller subsets of nodes. However, this interpretation is a posteriori in the following sense: Consider an open system, such as an infinite CA. Although locally only pair interaction takes place, as the system evolves in time we find higher order correlations. When we speak of higher order interactions here, we mean an interpretation of higher correlations by a model of higher order interaction in a closed system.

We look at examples for our quantities. Consider first a distribution $P$ which factors over the units, i.e. it has a representation

$$
P(x)=\prod_{v \in V} P_{v}\left(x_{v}\right),
$$

where $P_{v}$ are the marginals on single units. In this case $P$ is an element of the closure $\overline{\mathcal{E}_{1}}$ of the exponential family $\mathcal{E}_{1}$ and therefore $I^{(k)}(P)$ equals zero for $k \geq 2$.

For higher interactions a similar statement is true. One has that $P \in \overline{\mathcal{E}_{k}}$ if $P$ admits a factorization as

$$
P(x)=\prod_{A:|A|=k} \phi_{A}\left(x_{A}\right),
$$

while here the converse is not true. A given $P \in \overline{\mathcal{E}_{k}} \backslash \mathcal{E}_{k}$ need not have this structure. In (11) the functions $\phi_{A}$ have 
the property to depend on their argument only through the part in $A: x_{A}$. In this general case, the $\phi_{A}$ are not the marginals. If $P \in \overline{\mathcal{E}_{k}}$, then $I^{(l)}=0$ for $l>k$.

Consider next a distribution in the synchronized case, i.e. from the value of one unit $v$ all the other units are determined. Take as an example the distribution $P$ with $P(0 \cdots 0)=P(1 \cdots 1)=\frac{1}{2}$ and $P(x)=0$ for all other elements. It can be seen that these distributions lie in the closure of $\mathcal{E}_{2}$, since, loosely speaking, by pair interactions this behavior can be explained. To do so let the second, third,... unit copy the value of the first unit, then the synchronized behavior is "constructed" using only conditions on pairs. Since $P \in \overline{\mathcal{E}_{2}}$ we have that $D\left(P \| \mathcal{E}_{k}\right)=D\left(P \| \mathcal{E}_{2}\right)=0$ for all $k>2$ and therefore the only non vanishing component of $I(P)$ is $I^{(2)}$.

Another extreme case is given by the parity function. Let $P$ be the uniform distribution on the following set of elements

$$
\mathcal{Y}:=\left\{x: \mathcal{X}_{V}: x_{1}=\sum_{i=2}^{N} x_{i} \bmod 2\right\}
$$

$\mathcal{Y}$ is the set of all configurations with even parity. It can be seen that for this distribution $I^{(N)}$ is maximal while all other components vanish. This is due to the fact, that every choice of $N-1$ units in is independent under $P$, while there is total (functional) dependence between all the $N$ units. It can be seen that even the reverse statement is true. We elaborate a bit on this. Let $g$ : $\{0,1\}^{N-1} \rightarrow\{0,1\}$ be a binary function on the $N-1$ strings. Define the following set

$$
\mathcal{Y}_{g}:=\left\{x \in \mathcal{X}_{V}: x=(g(y), y) y \in\{0,1\}^{N-1}\right\}
$$

This means that $\mathcal{Y}_{g}$ is formed by those strings where the nodes $2, \ldots, N$ are chosen freely and the first node has the value of $g$. Let $P$ be the distribution that is uniform on the set $\mathcal{Y}_{g}$. Then it can be shown that if $I^{N}(P)>0$ then either $g$ is the parity function or $1+g \bmod 2$ is the parity function.

The vector $I$ has a natural correspondence to multiinformation (or integration as it is termed in [8]). Multiinformation is a generalization of mutual information and is defined as

$$
M(P):=\sum_{i \in V} H_{i}\left(P_{i}\right)-H(P),
$$

where $H(P):=-\sum_{x \in \mathcal{X}_{V}} P(x) \log P(x)$ is the entropy of $P$ and $P_{i}$ is the $\{i\}$-marginal of $P$. Then $H_{i}\left(P_{i}\right):=$ $-\sum_{x \in \mathcal{X}_{i}} P_{i}(x) \log P_{i}(x)$ is the marginal entropy in the unit $i$. Multi-information measures the distance of a distribution from independence of the units. It allows a decomposition as

$$
M(P)=D\left(P \| \mathcal{E}_{1}\right)=\sum_{k=2}^{N} I^{(k)}(P) .
$$

See [9] for these complexity measures, their properties, and the relation with TSE Complexity [8].

In [4], it is shown that the maximizer of multiinformation are contained in the closure of the exponential family $\mathcal{E}_{2}$ and therefore have the $I$-vector concentrated in $I^{(2)}$.

In the following we compare our approach with the one of [10]. There the author distinguishes a chemical contrast, which corresponds to our $I^{(1)}$. It quantifies the information that is contained in a sequence due to the average density of zeros differing from $\frac{1}{2}$. Then, quantities $k_{m}$, the correlational contrasts, were defined. These are in fact conditional mutual information, which measure the difference of the actual distribution to a Markov approximation. They also appear in the effective measure complexity [11] which can be expressed as a weighted sum over these quantities. The relation to our measures is that $k_{m}=0$ implies $I^{(m)}=0$. This can be understood from the fact that a distribution that has a Markov property with respect to $m-1$ marginals will be representable by $m-1$ interactions. On the other hand, a distribution that is representable by pair interactions does not necessarily admit a Markov representation as the example

$$
P(x)=\frac{1}{Z} \exp \left\{c_{1} x_{1} x_{2}+c_{2} x_{2} x_{3}+c_{3} x_{3} x_{1}\right\}
$$

of a triangle with no three-way interaction shows. Therefore our measures give a finer view onto the interaction structure.

Having identified "non complex" behaviors with extreme values of the components of $I(P)$ we now look for dynamical systems where $I$ is not concentrated in one component, because if several $I^{(k)}$ contribute, interactions on different scales coexist. This then could indicate complex behavior. We will next introduce our model systems where we try to find complex behavior in the above sense.

\section{MODELS AND METHODS}

\section{A. Model Systems \\ 1. Cellular Automata}

A possible natural choice for a model system are one dimensional (binary) cellular automata. They produce discrete output and are therefore ideally suited for our method. Furthermore, CA were introduced by S. Wolfram [12] as paradigmatic models to study complexity. In this work, we restrict ourselves to some of the 256 elementary binary CA studied by Wolfram [13]. For these, starting from a configuration $x^{(t)} \in\{0,1\}^{N}$ the next configuration $x^{(t+1)}$ at a site $i$ is computed as

$$
x_{i}^{(t+1)}=f\left(x_{i-1}^{(t)}, x_{i}^{(t)}, x_{i+1}^{(t)}\right)
$$

for some function $f:\{0,1\}^{3} \rightarrow\{0,1\}$. Typically one chooses periodic boundary conditions, i.e. $x_{N+1}$ is 
identified with $x_{1}$. There exist exactly 256 functions $\{0,1\}^{3} \rightarrow\{0,1\}$, each characterized by its binary vector of values in $\{0,1\}^{8}$. These vectors correspond to binary representations of integers smaller than 256 and the rules get numbered accordingly.

In this simple model, only the next neighbors of node $i$ influence this particular node. A priori, there are 88 elementary CAs modulo the inversion and reflection symmetry. Furthermore, there are trivial cases like rule 0, etc. such that the number of "interesting" rules is smaller. In this paper, we pick out some specific rules that have found attention in the literature. Specifically, we study the rules 18, 20, 22, 30, 45, 50, 90, 110, 126 and 150.

\section{Coupled Map Lattices}

Our second model system: Symbolic Dynamics of Coupled Map Lattices. Let again $V=\{1, \ldots, N\}$ denote the set of node indexes. Assume we are given a connection structure between the nodes, specified by a graph $G$ with vertices $V(G)=V$. By abuse of notation, the structure of the network is given by the symmetric adjacency matrix $G=\left(g_{i j}\right)_{i, j=1, \ldots, N} \in\{0,1\}^{N \times N}$ of the graph. We study the discrete time case $t=0,1,2, \ldots$ and every node $i \in V$ carries a real value $x_{i}(t) \in[0,1]$. These values get updated simultaneously according to the so called coupled tent map rule [14]

$$
x_{i}(t+1)=\epsilon \sum_{j} \frac{1}{k_{i}} g_{i j} f\left(x_{j}(t)\right)+(1-\epsilon) f\left(x_{i}(t)\right),
$$

where $k_{i}$ is the number of neighbors of node $v$ and $f$ : $[0,1] \rightarrow[0,1]$ defined by

$$
x \mapsto f(x)=\left\{\begin{array}{ll}
2 x & \text { if } 0 \leq x \leq \frac{1}{2} \\
2(1-x) & \text { if } \frac{1}{2} \leq x \leq 1
\end{array} .\right.
$$

is the tent map on the unit interval. Tent map lattices are a well established model to study synchronization and pattern formation in spatially extended systems [15, 16].

We will study binary symbolic dynamics of coupled tent map network [17, 18]. For each node we consider $\mathcal{X}_{i}:=\{0,1\}$ and for every $A \subseteq V$ we have $\mathcal{X}_{A}=\{0,1\}^{|A|}$ as in Section II. Then the configuration space of the symbolic dynamics is $\mathcal{X}_{V}=\{0,1\}^{N}$, the space of binary sequences of length $\mathrm{N}$. To every real valued dynamics $x_{i}(t)$ of node $i$, we consider the symbolic dynamics

$$
s_{i}(t)= \begin{cases}0 & \text { if } 0 \leq x_{i}(t) \leq x^{*} \\ 1 & \text { if } x^{*}<x_{i}(t) \leq 1\end{cases}
$$

where $x^{*} \in[0,1]$ is a given value. We obtain a global configuration of symbols $s(t)=\left(s_{1}(t), \ldots, s_{N}(t)\right) \in \mathcal{X}_{V}$. Our aim is to utilize this symbolic time-series in order to evaluate the complexity of the full dynamics. Assuming that a dynamical system has arrived in a stationary state it will produce a stationary distribution on the set of symbolic strings. The method we propose is to capture this stationary distribution $P$ and evaluate the complexity $I(P)$. We will call a dynamics complex if it exhibits interactions of different orders at the same time.

\section{B. Simulation and Computation}

\section{Cellular Automata}

As our computational power limits us to analyzing short sequences, we cut pieces of length 14 from CA of length 20000. These were iterated for $10^{6}$ time steps, starting from an i.i.d. initial condition. To gain more insight in the structure of our measures we furthermore evaluate it for the "interaction cone" corresponding to a single time step. To do so we take all binary strings of length 3 and compute the value of the $\mathrm{CA}$ on these strings. We then append the output as the 4th digit to the input string and compute $I^{(1)}, \ldots, I^{(4)}$ on the uniform distribution on these strings. Of course in the running CA one has to consider the influence of the stationary distribution on the inputs. To measure this influence we also evaluate the elementary 4-strings when the input is sampled with the stationary distribution.

\section{Coupled Map Lattice}

To simulate the coupled map lattice one has to specify a graph. For our simulations, we use a fully connected graph("globally coupled network"), and a circle graph ("next neighbor coupling") of 10 nodes each. We initialize the networks by setting the $x_{i}(0)$ to independently uniformly distributed random values in the real interval $(0,1)$. The dynamics generates two time-series, the real valued one $x(t)=\left(x_{1}(t), \ldots, x_{N}(t)\right)$ and, using $x^{*}=\frac{1}{2}$, the symbolic one $s(t)=\left(s_{1}(t), \ldots, s_{N}(t)\right)$. In the uncoupled case, the value $x^{*}=\frac{1}{2}$ gives the generating partition. It makes the symbolic time series most informative about the true time series. We discard the symbols during a transient time, assuming that after the transient time (chosen here as $10^{6}$ time steps), the system is in a stationary state. Treating the symbolic time-series as $N$ realizations of a random variable with probability distribution $P \in \overline{\mathcal{P}\left(\mathcal{X}_{V}\right)}$ on the space of symbols we compute $I(P)$ for this $P$.

We also experimented with different values of the bound $x^{*}$, but it turned out that this does not change the qualitative behavior. The value $x^{*}=\frac{2}{3}$ has received some attention as synchronization of the real valued dynamics can be detected from the symbols [19]. For our measures however, $I$ does not seem to depend on the choice of $x^{*}$ in such an essential manner. 


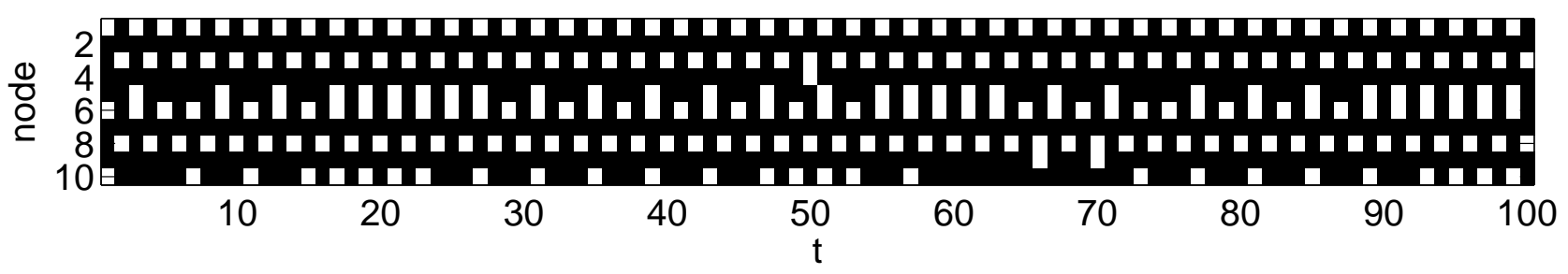

FIG. 1: A snapshot of the highly regular binary symbolic dynamics of the circle graph at $\epsilon=0.476$. Each row shows one node over time. White spots indicate the value zero, black squares one.

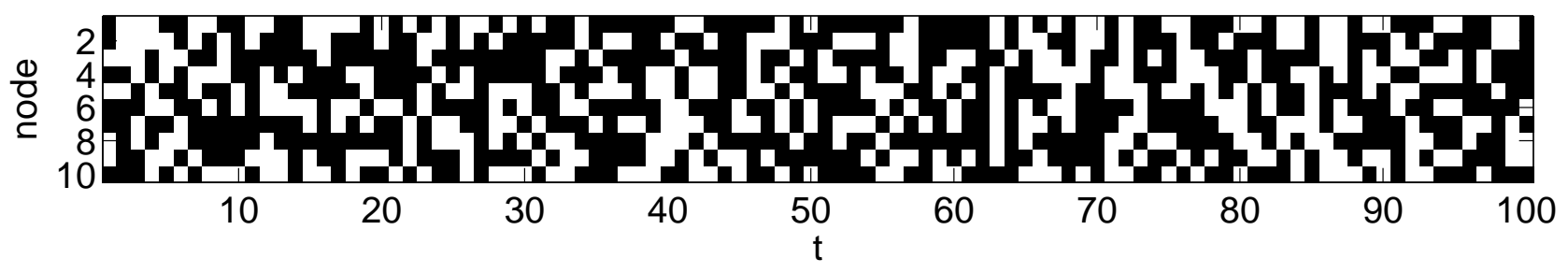

FIG. 2: A snapshot of the weakly coupled chaotic dynamic of the circle graph at $\epsilon=0.04$. Each row shows one node over time. White spots indicate the value zero, black squares one.

\section{Computation of $I^{(k)}$.}

As we have seen in Section $\amalg A$, computing an information projections, i.e. a minimizer of $D(P \| Q)$, for $P$ given and $Q \in \overline{\mathcal{E}}$ can be written as computing a maximum likelihood estimation in some discrete statistical model. This estimation can be solved analytically only for some very simple models, such as $\mathcal{E}_{1}$. Nevertheless, for the general case good iterative algorithms exist. These go back to Kullback [20, 21]. We have reimplemented the algorithm in parallel in the program CIPI which is freely available [22]. As the projections can be computed, the determination of $I^{(k)}$ is merely a subtraction of two KL-divergences.

\section{RESULTS}

A. Cellular Automata

In Table I the experimental values for sequences of length 14, cut from large cellular automata are given. Then in Tables $\Pi$ and $\amalg$ the values of the $I$-Vector for elementary steps of each of the automata are printed. We distinguish between the uniform input and the true input statistic sampled from the stationary distribution of the respective CA.

The values in Table $\prod r$ represent properties of the invariant measure of the respective CA. We confirm, that rules 30,45 , and 150 have the uniform distribution as their invariant measure, therefore no interaction can be detected. The results for rule 22 show, that the long range correlations, known from [23, 24], can be detected using our measure.

In the elementary interaction cone of $3+1$ elements,

\begin{tabular}{|c||c|c|c|c|c|c|c|c|}
\hline rule & $I^{(1)}$ & $I^{(2)}$ & $I^{(3)}$ & $I^{(4)}$ & $I^{(5)}$ & $I^{(6)}$ & $I^{(7)}$ & $I^{(8)}$ \\
\hline \hline 18 & 1.828 & 2.237 & 0.031 & 0.010 & 0.002 & 0 & 0 & 0 \\
\hline 20 & 1.890 & 1.556 & 0.256 & 0.028 & 0.003 & 0 & 0 & 0 \\
\hline 22 & 0.634 & 0.479 & 0.076 & 0.179 & 0.077 & 0.035 & 0.019 & 0.006 \\
\hline 30 & 0 & 0 & 0 & 0 & 0 & 0 & 0 & 0 \\
\hline 45 & 0 & 0 & 0 & 0 & 0 & 0 & 0 & 0 \\
\hline 50 & 0 & 9.011 & 0 & 0 & 0 & 0 & 0 & 0 \\
\hline 54 & 0.007 & 2.658 & 0.181 & 1.409 & 0.040 & 0.004 & 0 & 0 \\
\hline 90 & 0 & 0 & 0 & 0 & 0 & 0.002 & 0.002 & 0.002 \\
\hline 110 & 0.156 & 3.738 & 2.070 & 0.035 & 0.009 & 0 & 0 & 0 \\
\hline 126 & 0 & 2.809 & 0.307 & 0.095 & 0.004 & 0 & 0 & 0 \\
\hline 150 & 0 & 0 & 0 & 0 & 0 & 0 & 0 & 0 \\
\hline
\end{tabular}

TABLE I: Results for Cellular Automata, 0 stands for a value less than $10^{-3}$. $I^{(9)}$ to $I^{(14)}$ are not shown, as they vanish.

\begin{tabular}{|c||c|c|c|c|}
\hline rule & $I^{(1)}$ & $I^{(2)}$ & $I^{(3)}$ & $I^{(4)}$ \\
\hline \hline 18 & 0.189 & 0.311 & 0.500 & 0 \\
\hline 20 & 0.189 & 0.311 & 0.500 & 0 \\
\hline 22 & 0.046 & 0.158 & 0.800 & 0 \\
\hline 30 & 0 & 0.189 & 0.811 & 0 \\
\hline 45 & 0 & 0.189 & 0.811 & 0 \\
\hline 50 & 0.046 & 0.885 & 0.069 & 0 \\
\hline 54 & 0 & 0.189 & 0.811 & 0 \\
\hline 90 & 0 & 0 & 1 & 0 \\
\hline 110 & 0.046 & 0.158 & 0.796 & 0 \\
\hline 126 & 0.189 & 0 & 0.811 & 0 \\
\hline 150 & 0 & 0 & 0 & 1 \\
\hline
\end{tabular}

TABLE II: Cellular Automate $3 \rightarrow 1$ statistics with uniform inputs. 

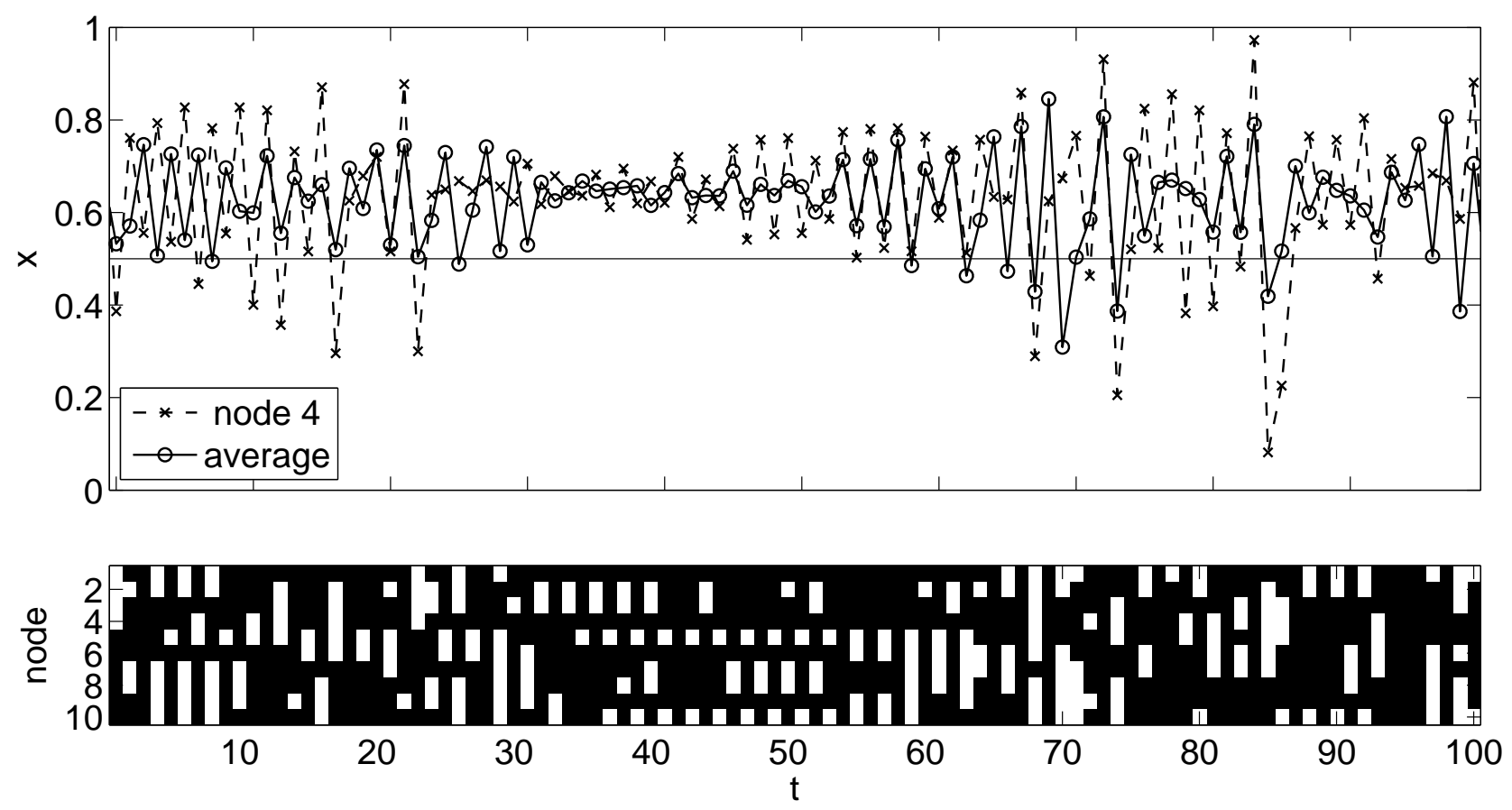

FIG. 3: A snapshot of the complex dynamic of the circle graph at $\epsilon=0.482$. In the top the activity of node 4 is plotted together with the average activity over all nodes. One can observe that for short periods of time synchronization effects occur, for instance between $t=30$ and $t=40$ node 4 is almost constant while the average also fluctuates less. Below the symbolic dynamics, with black as one, white as zero, of all 10 nodes is shown. The synchronization is visible here too as the pattern after $t=35$ is very regular.

\begin{tabular}{|c||c|c|c|c|}
\hline rule & $I^{(1)}$ & $I^{(2)}$ & $I^{(3)}$ & $I^{(4)}$ \\
\hline \hline 18 & 0.757 & 0.451 & 0.635 & 0 \\
\hline 20 & 0.822 & 0.403 & 0.535 & 0 \\
\hline 22 & 0.260 & 0.135 & 0.908 & 0 \\
\hline 30 & 0 & 0.189 & 0.811 & 0 \\
\hline 45 & 0 & 0.188 & 0.812 & 0 \\
\hline 50 & 0 & 3 & 0 & 0 \\
\hline 54 & 0.003 & 0.160 & 0.847 & 0 \\
\hline 90 & 0 & 0 & 1 & 0 \\
\hline 110 & 0.059 & 0.241 & 0.804 & 0 \\
\hline 126 & 0 & 0.500 & 1 & 0 \\
\hline 150 & 0 & 0 & 0 & 1 \\
\hline
\end{tabular}

TABLE III: Cellular Automate $3 \rightarrow 1$ statistics with specific input statistics.

we can observe the following principles: Rule 18 and rule 20 build up correlations in the input which leads to overall stronger correlations when the stationary distribution is used. One can also see, that the outputs are not uniformly distributed leading to a high value of $I^{(1)}$ when the inputs are sampled from the stationary distribution. In the elementary time step of rule 22 a lot of triple wise interactions are detected. This property is also found when the inputs are sampled from the stationary distribution. Rule 50 is a very simple rule which generates a periodic pattern of period 2. We see this represented here as (like in the synchronized case, described in the introduction) the $I$-vector is concentrated in $I_{2}$. When the inputs are uniform on the other hand, this rule has some small correlations of three positions, these get eliminated as the system reaches the stationary distribution. Rule 90 and rule 150 are additive rules that are modeled by the XOR function. Rule 150 takes XOR of every input, giving exactly the correlation between all 4 digits that was described in Section [II, Rule 90 is similar, it computes XOR of the inputs 1 and 3, leading to only triple wise interaction.

For rules $22,30,45$ there is evidence for the long range correlations in [25]. Here, we can distinguish rule 22 from the other two, as rule 30 and rule 45 still show the uniform distribution in the outputs leading to $I^{(1)}$ being zero. Rule 30, Stephen Wolfram's "all time favorite rule", is believed to have a high degree of randomness. (It might actually be used as a random number generator in Mathematica). For us it is not distinguishable from rule 45. Rule 110, which is capable of universal computation shows no specific behavior with respect to our measure. Rule 126 has a mirror symmetry leading to $I^{(2)}$ being zero when the input is sampled uniformly. However, the stationary distribution induces a different symmetry. This leads to an interesting statistics, which 
looks as follows

$$
p_{126}(x)= \begin{cases}\frac{1}{8} & \text { for } x \in\{(001),(011),(100),(110)\} \\ \frac{1}{4} & \text { for } x \in\{(000),(111)\} \\ 0 & \text { otherwise }\end{cases}
$$

It can be seen that the rule exactly computes XOR of input 1 and 3 on these configurations (which it does not do on all configurations). This leads to the contribution of $I^{(3)}$. On the other hand, the nonexistence of the configurations (101) and (010) can only be accomplished by additional interactions of order 2. A comparison with rule 90 shows the non-additive nature of $I$. The exactness of the probabilities accounts for the exact values in $I$. In fact these values can be predicted from theory.

\section{B. Coupled Tent Maps}

\section{Fully connected Graph}
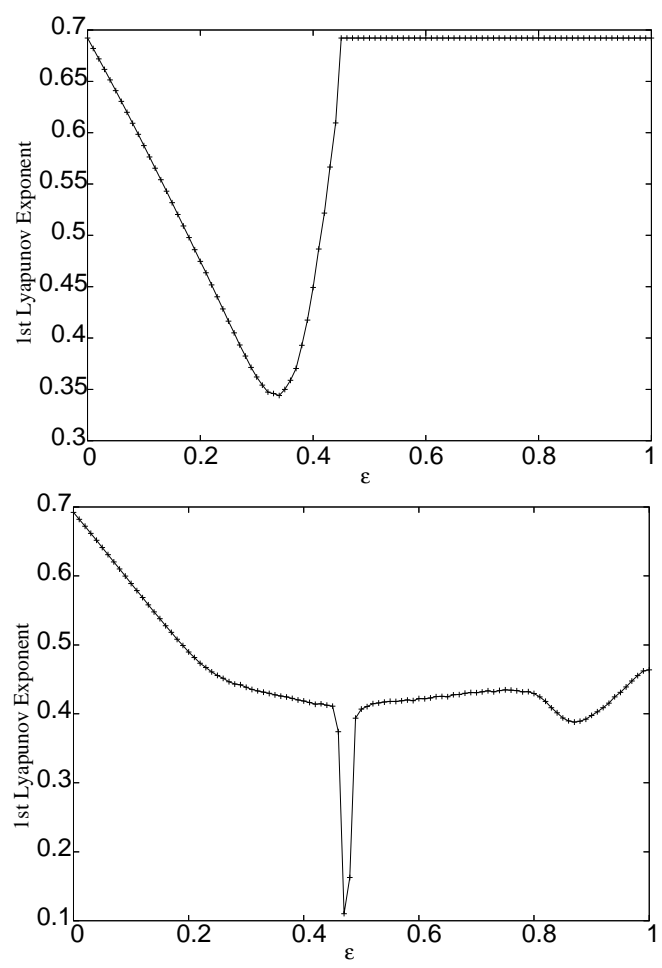

FIG. 4: Behavior of the largest Lyapunov exponent $\lambda_{1}$ for the fully connected graph(top), and the circle graph(bottom). Note that the minima are attained in exactly the same parameter region, in which the $I^{(k)}$ have maxima.

In Figures 5 and 6 the values of $I^{(1)}$ to $I^{(6)}$ are plotted for the fully connected and the circle graph, depending on the coupling $\epsilon$. For $k>6$, the $I^{(k)}$ are very small and depend strongly on the random initial condition, so that we don't evaluate them here. Figure 4 shows the behavior of the largest Lyapunov exponent for the two network structures. Different snapshots of the symbolic dynamics of the circle graph are given in Figures 1, 2, and 3 .

For the fully connected graph, the nodes will be driven to synchronized chaos if the coupling strength is high enough [26, 27]. We see this here as for $\epsilon>0.45 I$ is concentrated in $I^{(2)}$. It is interesting to observe that the $I^{(k)}$ indicate complex dynamics taking place on the edge of synchronized regimes. The largest Lyapunov exponent becomes smallest at $\epsilon=0.34$ (but not zero or negative, which would indicate periodicity). The respective maxima of the $I^{(k)}$ correspond to this. They are located at $\epsilon_{3}=0.345, \epsilon_{4}=0.42, \epsilon_{5}=0.355, \epsilon_{6}=0.35$. Also note that $I^{(4)}$ has at least a local maximum at the parameter value 0.35 .

\section{Circle Graph}

The circle graph of 10 nodes shows a specific "nearly periodic" behavior for parameter values $\epsilon \in$ $[0.463,0.481]$. This is a specific feature due to the symmetry of the system. It is not present for $N \neq 10$. We don't want to call the dynamics in that regime complex, as there is a high degree of regularity. In the respective parameter region, the average activity taken over all nodes is almost constant. In the symbolic dynamics, 2 of the nodes are constant themselves, 4 are periodic, while the remaining 4 are close to periodicity(see Figure 1). Obviously, the complexity measures detect this phenomenon, as all $I^{(k)}, k \neq 2$ drop to zero. More interestingly, when the parameter $\epsilon$ takes values close to where the regularity occurs we can observe very high values of the $I^{(k)}$ for $k \neq 2$. In this regime, the average activity does not follow the one of the individual nodes, but fluctuates on the same scale, instead of averaging out. This indicates a complex dynamical structure driven by higher order correlations among the nodes. See Figure 3 for a snapshot of the dynamics. On the onset of the synchronization one can additionally observe "part time synchronization". This means that a nearly periodic state, as described above, emerges, but is not stable. After a couple of time steps (depending on the exact parameter value), it dissolves again.

To understand the reason for the peak close to the periodic phenomenon better we investigated the transition region closer. First, we took a very long sequence that shows transitions. Then we separated the two phases, saving them to different files and analyzed them separately. The result here is, that the unordered state shows the same $I$ vector as the region left of the peak, while the periodic sequences of course have $I$ concentrated in $I^{(2)}$, as the theory predicts. If the two types of sequences are mixed then higher order correlations appear, leading to the peak. This corresponds to the more general and unsolved problem whether the complexity of a convex combination of two distributions is related to the complexities of the individual constituents. 

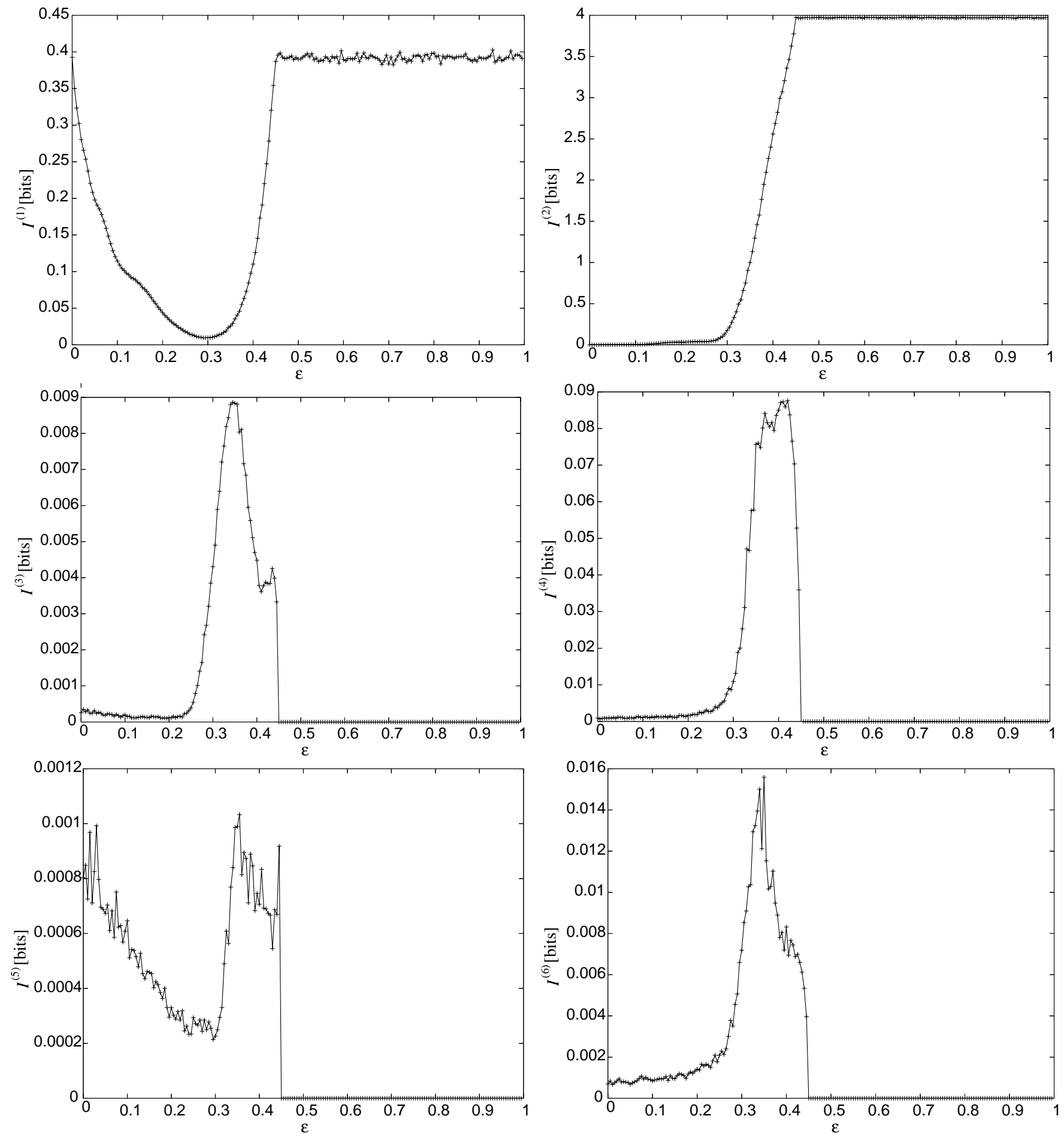

FIG. 5: The Values of $I^{(k)}$ for the fully connected graph. For $\epsilon>0.45$, the network is in the state of synchronized chaos. This is fully captured by pair interactions, therefore $I^{(2)}$ is maximal, while the $I^{(k)}$ for $k>2$ vanish. On the edge to synchronized chaos high correlations on different scales are detected.

\section{CONCLUSION}

We have introduced complexity measures, suggested by [1, 9], that quantify the interactions of $k$ parts of a system that cannot be explained by interaction between fewer parts of that system. Then, after studying elementary theoretical properties and their interpretation, we have explored the measures in numerical studies. Using the elementary cellular automata one can already study the behavior of the measures in special cases. There are 

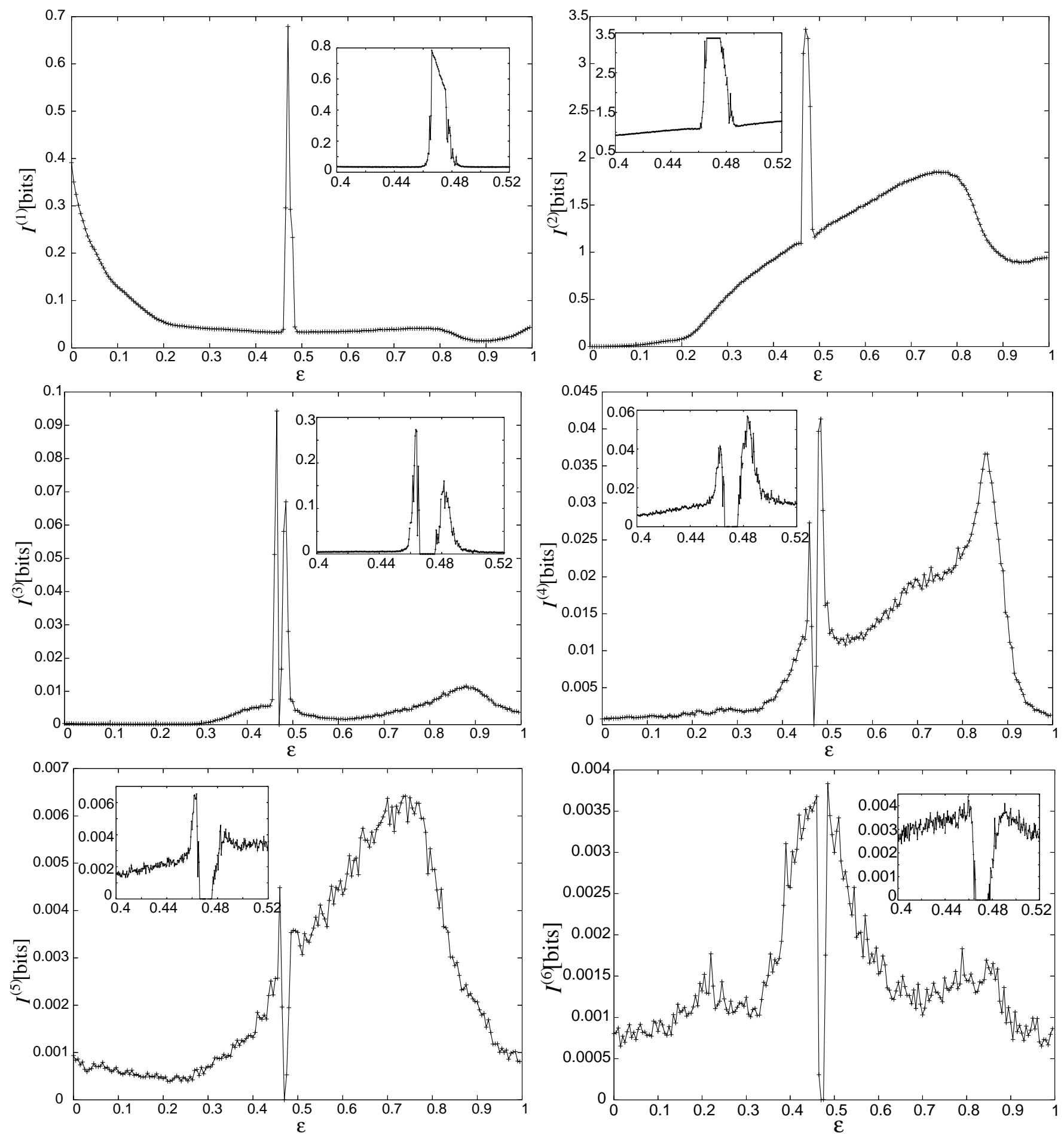

FIG. 6: The values of $I^{(k)}$ for the circle graph. The inlays show the parameter region $\epsilon \in[0.46,0.49]$.

specific effects, such as emergence of higher correlations, that can be found with our method. Commonly studied quantities like correlation functions capture only part of what we called $I^{(2)}$. Our measures therefore give a more global view.

Symbolic dynamics of coupled map lattices are far less understood from a theoretical point of view. We have shown that using the symbolic dynamics one can detect important dynamical properties of the underlying real valued dynamics. This is true despite the fact that only spatial correlations are used in the computation of the $I^{(k)}$. We stress the point that our measures take as input only the statistics of the system and make no model assumptions. They are suitable for exploratory analysis, as 
it is done in sequence based genetics. In an exploratory study one has no order parameters, as these quantities need to be constructed from some model first.

Interestingly, we observed that complex behavior takes place on the edge of synchronization, similar to the common wisdom of "complex dynamics on the edge of chaos". $I^{(2)}$ plays a special role in this context. As synchronization phenomena are entirely captured by pair interactions, we would suggest to measure complexity in terms of $I^{(k)}$ for $k>2$. There is more work to do, investigating this notion of complexity in different kinds of models.

Nevertheless the universality of the results remains to be investigated, as our system size is very small and the computation of the $I^{(k)}$ gets infeasible as the system grows. Therefore, for the time being our approach is practically limited, while still conceptually appealing.

\section{Acknowledgments}

The authors wish to thank Sarika Jalan for discussions in the early stages of the work. This work is funded by the Volkswagen Foundation.
[1] S. Amari, IEEE Transactions on Information Theory 47, 1701 (2001).

[2] S. Amari, Methods of Information Geometry, vol. 191 of Translations of mathematical Monographs (American Mathematical Society, 2000).

[3] J. N. Darroch, S. L. Lauritzen, and T. P. Speed, The Annals of Statistics 8, 522 (1980).

[4] N. Ay and A. Knauf, Kybernetika 42, 517 (2006).

[5] I. Csiszár and F. Matúš, in Proceedings on IEEE International Symposium on Information Theory 2002 (2002), p. 434.

[6] T. Kahle, W. Wenzel, and N. Ay, Kybernetika (2008), submitted.

[7] I. Csiszár and P. C. Shields, Information Theory and Statistics: A Tutorial, Foundations and Trends in Communications and Information Theory (now Publishers, 2004), URL http://www.renyi.hu/ csiszar/Publications/Informat

[8] G. Tononi, O. Sporns, and M. Edelman, Proc. Natl. Acad. Sci. USA 91, 5033 (1994).

[9] N. Ay, E. Olbrich, N. Bertschinger, and J. Jost, in Proceedings of the ECCS'06 (2006).

[10] K. Lindgren, Complex Systems 1, 592 (1987).

[11] P. Grassberger, Int. J. Theor. Phys. 25, 907 (1986).

[12] S. Wolfram, Reviews of Modern Physics 55, 601 (1983).

[13] S. Wolfram, Cellular Automata and Complexity
(Addison-Wesley, 1994).

[14] K. Kaneko, Chaos 2, 279 (1992).

[15] M. Droz and A. Lipowski, Phys. Rev. E 67, 056204 (2003).

[16] C. Hauptmann, H. Touchette, and M. C. Mackey, Phys. Rev. E 67, 026217 (2003).

[17] D. Lind and B. Marcus, Symbolic dynamics and coding (Cambridge University Press, 1995).

[18] S. Jalan, J. Jost, and F. M. Atay, Chaos 16, 033124 (2006).

[19] S. Jalan, F. M. Atay, and J. Jost, arXiv:nlin.CD/0510057.

[20] S. Kullback, Information Theory and Statistics (Dover, New York, 1968).

[21] I. Csiszár, Ann. Probab. 3, 146 (1975).

[22] L. Steiner and T. Kahle, cipi-computing information projections iterativly, Available at

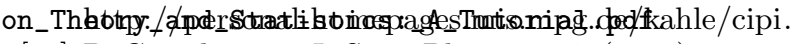

23] P. Grassberger, J. Stat. Phys 45, 27 (1986).

[24] P. Grassberger, Phys. Rev. E 59, R2520 (1999).

[25] P. Grassberger, J. Phys. A: Math. Gen. 20, 4039 (1987).

[26] K. Kaneko, Physica D 41, 137 (1990).

[27] R. E. Amritkar, S. Jalan, and C. K. Hu, Phys. Rev. E 72, 016212 (2005). 